\title{
Empowerment braucht Change-Management
}

VON BEATE BLANK

Dr. Beate Blank leitet das Beratungsbüro »empowerment« in Stuttgart. Sie bietet Empowerment Beratung und Coaching an. Zudem führt sie Forschungsprojekte und Weiterbildungsveranstaltungen zu diesem Thema durch.

http://empowermentconsulting.de

\author{
Das Konzept des Empowerments, der \\ "Selbstbefähigung" zielt darauf ab, die Autonomie \\ und die Eigenmacht der Nutzer zu stärken. Die darauf \\ basierenden Arbeitsansätze wollen vorhandene, \\ wenngleich vielleicht verschüttete Fähigkeiten \\ der Klientinnen und Klienten stärken. In der Praxis \\ von sozialen Organisationen und Unternehmen \\ stößt diese Absicht - bei aller theoretischen \\ Zustimmung - doch oft an ihre Grenzen.
}

Im Zuge des aktuellen gesellschaftlichen Diskurses um Inklusion, Integration, Teilhabe und Verwirklichungschancen rückt das internationale Empowerment-Konzept wieder in den Fokus der Fachöffentlichkeit.

Mit Blick auf die Herausforderungen der Globalisierung gilt den Theoretikern der Empowerment-Ansatz als »ein besonders zukunftsweisendes Modell in der Bewältigung der komplexen Aufgaben Sozialer Arbeit im Kontext gesellschaftlicher Veränderungsprozesse« (Pankofer 2000:7) »Ein sehr hoher Anspruch würde ich sagen «, so Silvia Staub-Bernasconi, "aber, da ich selbst vom >Ende der Bescheidenheit rede, geht es mir mehr darum, herauszufinden, wie er eingelöst wird « (Staub-Bernasconi 2007:247).

Mit der These der Interdependenz von Ressourcenförderung und Empowerment (Blank 2012) habe ich die in meiner Beratungspraxis oft gestellte Wie-Frage im Theorie-Praxis-Bezug untersucht und nach ihrer Anschlussfähigkeit im Diskurs Sozialer Arbeit gefragt. Es war Barbara B. Solomon (1976), die den Empowerment-Begriff in den Diskurs Sozialer Arbeit eingeführt und im Sinne eines »Empowerment verhindernden oder ermöglichenden Hilfesystems " machttheoretisch begründet hat.

\section{Die Empowerment-Kritik}

Dem hohen normativen und politischen Anspruch steht fundamentale Kritik gegenüber. Dies hat auch mit der historischen Rezeption und breiten disziplinären Ausgestaltung der EmpowermentIdee zu tun. Sie reicht von psychosozial begründeten Zugängen (Rappaport u. a.1984, Stark 1996), über betriebswirtschaftliche Managementstrategien (Blanchard u. a.1999), bis hin zu methodischen Ausdifferenzierungen und Modernisierungen der Hilfe zur Selbsthilfe durch Soziale Arbeit (Herriger 1999).

Was Wunder, dass der Empowerment-Begriff in dieser Breite als kaum fassbar und schillernd erscheint. Empowerment-Kritiker monieren zu Recht, dass die theoretische Ungenauigkeit einer unzulässigen Individualisierung und neoliberalen Vereinnahmung des ursprünglich der emanzipatorischen Idee verpflichteten Ansatzes Vorschub leiste. »Wo bleibt die Macht? « (StaubBernasconi 2007:249 ff.) ist die meist gestellte kritische Anfrage an das Empowerment-Konzept.

\section{Die Machtfrage}

Trotz aller Versuche einer individualisierenden Engführung ist die Machtfrage im Empowerment-Konzept inhärent. Im Englischen »power« potenzieren sich in 
der Übersetzung gleich drei Begriffe: Kraft, Stärke, Macht.

Die Machtfrage an sich ist auch ohne Machtanspruch der Adressatinnen und Adressaten eine Herausforderung an das Hilfesystem und seine Organisationen. Die Globalisierung macht vor nationalen, sozialstaatlich begründeten Begrenzungen nicht Halt und stellt die Träger unter einen erheblichen Druck, Rechtsansprüche nach Teilhabe ihrer Adressaten in ihre Konzept- und Organisationsentwicklung mit einzubeziehen. Wie sollen künftig sozialstaatliche Erfordernisse und demokratische Rechtsstaatlichkeit verbunden werden mit der Gerechtigkeitsfrage, mit ethischen Grundwerten und dem Teilhabepostulat?

In der Sozialen Arbeit und im Gesundheitswesen können wir gelingende Empowerment-Prozesse auf personaler und zunehmend auf sozialer Ebene sehen. Doch sobald die Frage der Implementierung von erfolgreichen Projekten in die alltägliche Arbeit der Institutionen ansteht, werden die greifbaren positiven Ergebnisse problematisiert: Wie würde die strukturelle Verankerung der Partizipation der Adressaten unsere Arbeit verändern? Ist ihr Beteiligungsanspruch mit unserem Auftrag der Hilfe und Kontrolle vereinbar? Wie gehen wir mit ihren gestiegenen Erwartungen um? Treten wir damit nicht etwas los, das wir nicht mehr kontrollieren können?

Diese Befürchtungen sind berechtigte Anfragen an das EmpowermentKonzept. Sie machen deutlich, dass Empowerment-Prozesse gleichsam natürlicherweise die Machtfrage berühren und von innen heraus, beabsichtigt oder unbeabsichtigt, zum Change Management führen.

\section{Die komplexe Dynamik}

Empowerment-Prozesse lösen zirkuläre Dynamiken auf vier sozialstrukturellen Ebenen aus:

- auf der personalen Ebene: Autonomie, Einzigartigkeit und Vielfalt des Individuums

- auf der sozialen Ebene: Peer Groups, Selbstorganisationen

- auf der der organisationalen Ebene: Institutionen, Projekte, intermediäre Räume sozialer Stadtentwicklung)
- auf der gesellschaftlichen Ebene: Hilfesystem, Nationalstaat, Europäische Union

Alle vier werden systemisch vernetzt gedacht und strategisch als gemeinsame Konstruktion einer Ko-Produktion zwischen Adressaten und Hilfesystem im Sinne einer lernenden Gemeinschaft transparent gesteuert. Der systemischkonstruktivistische Ansatz von Organisationsentwicklung wird im Verständnis von Macht- und Ressourcenungleichkeit in Empowerment-Prozessen konkretisiert. Besonders berücksichtigt werden die selbstermächtigenden Wirkungen, die wegen ihrer Eigendynamik bei den Verantwortlichen zu erheblichen Verunsicherungen führen können. »Jedes System organisiert vor allem anderen seine Selbsterhaltung«, so Niklas Luhmann. Diesen Grundsatz gilt es in den komplex wirkenden Dynamiken von Empowerment-Prozessen anzuerkennen und zu steuern.

\section{Die Ressourcen(um)verteilung}

Im Prozess der Neubesinnung im Hilfeund Interventionsverständnis können Managementstrategien hilfreich sein, die Selbsterhaltung (Begrenzung) und Teilhabe (Entgrenzung) verbinden. Das Instrument »Partizipatives Ressorucenmanagement « (Blank 2007) setzt die Teilhabe der Nutzer auf den vier Empowerment-Ebenen um. Da beispielsweise alle an den Ressourcen des Quartiers teilhaben können, wird ein großer Nutzen für die beteiligten Akteursgruppen generiert. Das Akteursund Erneuerungsprinzip bleibt, neben der Sicherheit des Zugewinns durch Reziprozität, gewahrt. Denn das intrinstisch motivierte Streben jedes Einzelnen nach Selbstbestimmung, Teilhabe, Anerkennung und gelingendem Leben ist konstitutiv für personale, soziale, organisationale und gesellschaftliche Ermächtigungsprozesse.

Die Solidarität, Zugehörigkeit und Sicherheit vermittelnde Peer Group erfährt, im Vergleich zur Ressourcenausstattung der Projektträger, noch wenig Pflege und erhält kaum Mittel, um nach Projektende den Adressaten weiterhin als Ressource zur Verfügung stehen zu können. An dieser Stelle erleben wir vonseiten der Träger häufig eine funktionalisierte Sicht auf das Empowerment-
Konzept: Es hat für rege Beteiligung, neue Perspektiven, für Öffentlichkeit und Reputation gesorgt und damit seine Aufgabe erfüllt. So gestärkt entscheiden die Verantwortlichen gerne, dass es nun die Aufgabe der »Empowerten « sei, weiterhin ehrenamtlich und ohne die bisher vorhandenen Ressourcen für ihre Teilhabe zu sorgen.

\section{Resümee: Die Ko-Produktion hilft allen}

Unterstützt wird die Fokussierung auf kurzfristigen Erfolg durch die Kurzlebigkeit der Förderprogramme. Sie berücksichtigen nicht die zirkuläre Wirkung von Selbstermächtigungs- und Beteiligungsprozessen. Aus Perspektive des Empowerment-Konzepts ist dies ein schwerwiegender Steuerungsfehler. Denn dadurch geht wertvolles Empowerment-Wissen verloren, das dringend für die Einlösung von Verwirklichungschancen ressourcenarmer Personen und Gruppen gebraucht wird.

Organisationen haben mehr Vorals Nachteile, wenn sie sich den durch Empowerment-Prozesse ausgelösten Herausforderungen stellen. Durch sie wird unverzichtbares soziales und kulturelles Kapital (Bourdieu 1983) generiert. Scheinbar nebenbei lernen die beteiligten Personen und Gruppen - im gemeinsamen Interesse - miteinander zu kooperieren und Zukunft auf der Grundlage ihrer wachsenden Reziprozität zu gestalten.

Zurzeit können wir im Zuge des Rechtsanspruchs auf Inklusion erste Konturen organisationaler Gestaltung von Empowerment-Prozessen beobachten. Hier wird deutlich, dass das Empowerment-Konzept ein geeigneter Handlungsrahmen für die Umsetzung der Teilhabe alle ist, von der personalen bis zur gesellschaftlichen Ebene.

\section{Literatur}

Blank, Beate (2012): Die Interdependenz von Ressourcenförderung und Empowerment. Der Ressourcenbegriff der AdressatInnen. Opladen. Solomon, Barbara B. (1976): Black Empowerment. Social Work in Oppressed Communities. New York.

Staub-Bernasconi, Silvia (2007): Soziale Arbeit als Handlungswissenschaft. Weinheim. 